\title{
Main constituents of polyphenol complex from seagrasses of the genus Zostera, their antidiabetic properties and mechanisms of action (Review)
}

\author{
OLGA NIKOLAEVNA STYSHOVA ${ }^{1}$, ALEXANDER MICHAILOVICH POPOV ${ }^{1,2}$, \\ ALEXANDER ALEKSEEVISH ARTYUKOV ${ }^{1}$ and ANNA ANATOLIEVNA KLIMOVICH ${ }^{1}$
}

${ }^{1}$ G.B. Elyakov Pacific Institute of Bioorganic Chemistry, Far Eastern Branch, Russian Academy of Sciences,
Vladivostok 690022; ${ }^{2}$ School of Natural Sciences, Far Eastern Federal University, Vladivostok 690000, Russia

Received June 3, 2016; Accepted November 11, 2016

DOI: $10.3892 /$ etm.2017.4217

\begin{abstract}
The present review analyzed the recent experimental studies of the alleviating activity of main constituents of the polyphenol complex from seagrasses of the genus Zostera, namely rosmarinic acid, luteolin and its sulfated derivatives, on carbohydrate and lipid metabolism disorders. A number of studies by our group and others, in which various experimental models of diabetes and hyperlipidemia were used, show a therapeutic action of the polyphenol complex and the abovementioned phenolic constituents, when applied separately and in combination. Based on the analysis of the results of these studies, the probable mechanisms of the therapeutic action of these compounds in diabetes and hyperlipidemia were proposed.
\end{abstract}

Correspondence to: Dr Olga Nikolaevna Styshova, G.B. Elyakov Pacific Institute of Bioorganic Chemistry, Far Eastern Branch, Russian Academy of Sciences, pr-t 100 let Vladivostoku 159, Vladivostok 690022, Russia

E-mail: krivoshapkoon@mail.ru

Abbreviations: T2D, type 2 diabetes; MS, metabolic syndrome; BAS, biologically active substances; PPC, polyphenol complex; RA, rosmarinic acid; LT, luteolin; DSL, 7,3'-disulfate luteolin; PPARs, peroxisome proliferator-activated receptors; FFAs, free fatty acids; TZDs, thiazolidinediones; LP, lipid peroxidation; ROS, reactive oxygen species; Nrf2, nuclear factor erythroid-2 p45-related factor 2; NO, nitric oxide; iNOS, inducible nitric oxide synthase; cPLA2, cytosolic phospholipase A2; LPS, lipopolysaccharide; TLR4, Toll-like receptor 4; NF- $\kappa \mathrm{B}$, nuclear factor $\kappa \mathrm{B}$; JNK, c-Jun $\mathrm{N}$-terminal kinases; TNF- $\alpha$, tumor necrosis factor alpha; IL, interleukin; SR, scavenger receptor; oxLDL, oxidized low-density lipoprotein; NSAID, non-steroidal anti-inflammatory agent; SAID, steroidal anti-inflammatory agent; PKC, protein kinase C; PTC, protein tyrosine kinase; MAPK, mitogen-activated protein kinase; COX, cyclooxygenase; LOX, lipoxygenase

Key words: metabolic syndrome, diabetes, hyperlipidemia, flavonoids, luteolin, rosmarinic acid

\section{Contents}

1. Introduction

2. Proposed mechanisms of the pharmacologic activity of RA and LT as well as its analogues

3. Antiinflammatory and antioxidant activity of RA, LT and its analogues

4. Features of the antidiabetic action of DSL

5. Conclusions

\section{Introduction}

In recent years, a large number of studies have focused on disorders of lipid and carbohydrate metabolism as the etiologic factors of type 2 diabetes (T2D) and atherosclerosis, and to be associated with their progression. Millions of people are suffering from metabolic syndrome and cardiovascular failure worldwide, which are considered the most common causes of human mortality $(1,2)$. The concept of 'metabolic syndrome' (MS) (2), which indicates a close association between certain types of hyperlipidemia and impaired glucose tolerance, has been widely accepted. One of the most important tasks for modern medicine is to identify novel, effective and easily obtainable remedies with low toxicity for preventive and adjunctive therapy of T2D and hyperlipidemia among natural sources of biologically active substances (BAS), which possess various mechanisms of protective action, aimed at restoring the disturbed biochemical status of the body (3-7).

Marine organisms are a rich source of natural products. To date, $>20$ million low-molecular-weight natural marine BASs, which often differ from secondary metabolites of terrestrial organisms in terms of chemical structure as well as in their biological effects, have been isolated and identified. The variety of chemical structures, the high biological activity and the prospects for their effective therapeutic application are the factors that attract increasing attention to substances of marine origin (4). It should be noted that certain major active components, i.e. rosmarinic acid and luteolin, have been originally found in terrestrial plants, such as rosemary and artichokes, 
while the disulfated derivative of luteolin may be of specific marine origin, but is a metabolic product of luteolin in the body (5-7).

The present review performed a brief analysis of published experimental data by our and other groups and postulated the possible mechanisms of the antidiabetic effects of the polyphenol complex (PPC) isolated from seagrasses of the genus Zostera (Z.; Z. marina and Z. asiatica), and its main components, namely rosmarinic acid (RA), luteolin (LT) and its disulfated derivative, 7,3'-disulfate luteolin (DSL), whose chemical structures are presented in Fig. 1.

The chemical composition of PPC from seagrasses of the genus $Z$. was analyzed by using high-performance liquid chromatography/mass spectrometry (Fig. 2).

As shown in Fig. 2, a major portion of the PPC is comprised of RA (45\%) and mono- and disulfated derivatives of LT ( 45\%); bioflavonoids including LT and apigenin constitute the remaining portion. The Laboratory of Biotechnology at the G.B. Elyakov Pacific Institute of Bioorganic Chemistry, Far Eastern Branch of the Russian Academy of Sciences (Vladivostok, Russia) has developed a method of isolating DSL (Fig. 2) from seagrasses of the genus $Z$. and performed extensive pharmacological trials in experimental animal models to compare its activities to those of the flavone LT and other commercial pharmaceuticals (8-10).

As recent studies on the antidiabetic properties of the main constituents of the PPC, such as RA and LT, have made significant progress, the present review focused on analyzing their results to postulate possible mechanisms of action. In addition, the present study attempted to elucidate why DSL has higher activity than LT with regard to a number of pharmacological properties. This information will be useful for the development of more effective DSL-based remedies for adjunctive therapy of MS and T2D.

\section{Proposed mechanisms of the pharmacologic activity of RA and LT as well as its analogues}

Effect exerted on peroxisome proliferator-activated receptor (PPAR) signaling pathways. According to studies by our group using experimental animal models of hyperglycemia (model of alloxan-induced diabetes) and hyperlipidemia (tyloxapol model), the PPC exerted potent antidiabetic and antihyperlipidemic effects leading to substantial normalization of clinical blood plasma parameters compared to those in untreated animals. In most cases, the therapeutic effect of the entire complex and its individual components proved to be stronger than that of known commercial pharmaceutical drugs applied for treating MS-associated pathologies (glibenclamide, metformin and nicotinic acid), which were used as positive controls (5-7). The high therapeutic activity of the PPC is undoubtedly determined by the pharmacological properties of its main components RA, LT and DSL, the biological activity and the possible mechanisms of action of which are discussed below.

It has been established that the phenylpropanoid RA, the flavone LT and its analogues exert pronounced antioxidant and anti-inflammatory effects, and have modulating effects on PPARs, which are among the main regulators of lipid and carbohydrate metabolism $(5,6,11,12)$.<smiles>O=C(/C=C\c1ccc(O)c(O)c1)OCC(C(=O)O)c1ccc(O)c(O)c1</smiles>

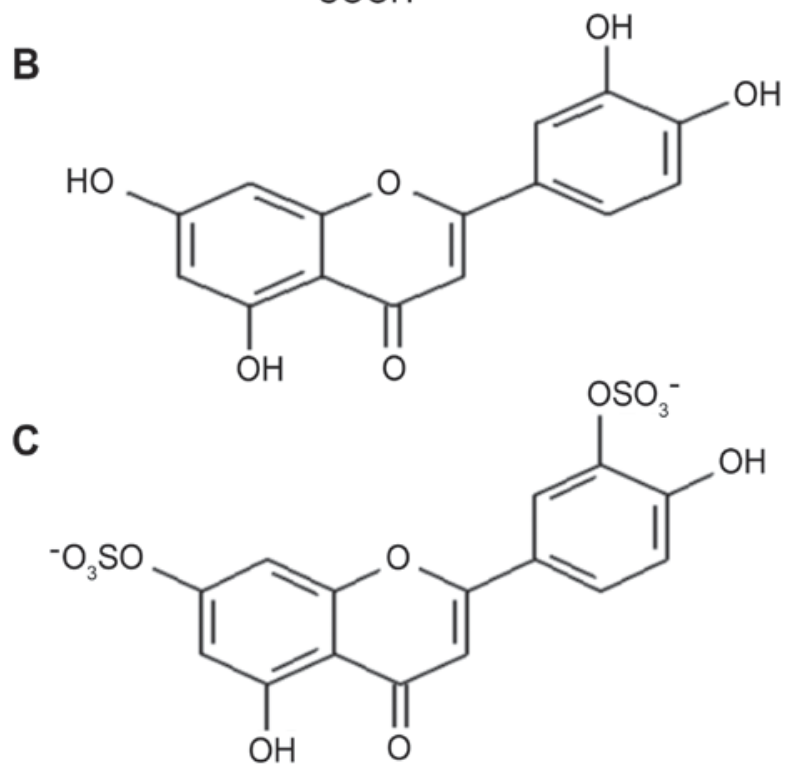

Figure 1. Chemical structures of main components of polyphenol complex from seagrasses of the genus Zostera. (A) rosmarinic acid, (B) luteolin and (C) 7,3'-disulfate luteolin.

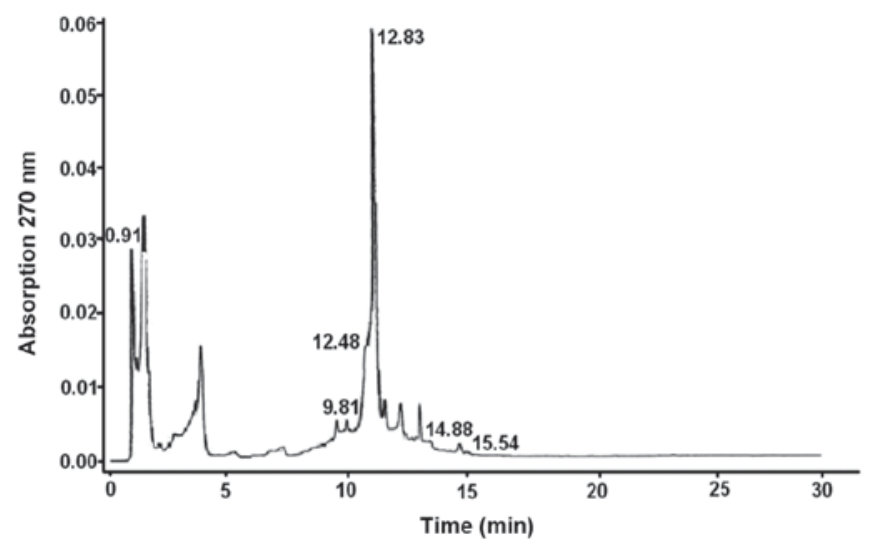

Figure 2. High-performance liquid chromatography/mass spectrometry spectrum of polyphenol complex. Retention times (min): 12.48, 7,3'-disulfate luteolin; 12.83, rosmarinic acid; 14.88, luteolin; 15.54, apigenin.

PPAR has several known isoforms: PPAR $\alpha, \operatorname{PPAR} \gamma$ (subtypes 1 and 2) as well as PPAR $\beta$ and PPARd, which are all cell receptors with a key role in preventing the pathologies associated with the occurrence and progression of T2D and MS. PPARs are nuclear receptors that regulate transcriptional activity; they can be modulated by binding of a large variety of small ligands, including certain phenolic compounds. PPARs have crucial roles as molecular sensors of environmental factors, including nutritional constituents to 'fine-tune' lipid 
and glucose homeostasis, and are also important for other physiological processes. Subsequent to activation, PPARs may form heterodimers with retinoid $\mathrm{X}$ receptors, which bind to the PPAR response elements in the promoter regions of expressed genes and either increase or reduce their transcription, the main effect of which being an increase in the storage of fatty acids in adipocytes, while the amount of fatty acids present in the circulation is decreased $(4-7,13,14)$.

PPAR $\gamma$ is predominantly expressed in adipose tissue. The endogenous ligands for these receptors are free fatty acids (FFAs) and a few of their exogenous ligands are used as therapeutic agents for the treatment of T2D. Antagonists of PPAR $\gamma$ prevent obesity and exert antidiabetic activity. Furthermore, they reduce adhesion of monocytes to endothelial cells and inhibit the inflammatory action of macrophages. Activation of PPAR $\gamma$ alters the expression of genes involved in metabolic processes such as adipogenesis, insulin signal transmission and glucose transport, resulting in the reduction of the respective tissue's resistance to insulin. RA and LT are PPAR $\gamma$ ligands with antioxidant and anti-inflammatory properties $(4-7,12,13,15)$.

Various studies have suggested that RA can function as a PPAR $\gamma$ antagonist by inhibiting adipogenesis and PPAR $\gamma$-dependent gene expression (12), while LT can either function as a PPAR $\gamma$ agonist by enhancing the expression of PPAR $\gamma$-dependent genes in adipocytes (16) or a PPAR $\gamma$ antagonist $(12,17)$. LT binds PPAR $\gamma$, but unlike thiazolidinediones (TZDs), which are a class of modern antidiabetic and antihyperlipidemic medicinal drugs, it does not promote adipocyte differentiation. LT exhibits mild partial agonist/antagonist activity, inhibits several PPAR $\gamma$ target genes and PPAR $\gamma$-dependent adipogenesis, but activates the glucose transporter type 4 (GLUT4) to a similar degree to that of RA, implying gene-specific partial agonism. LT is thought to be useful as a safe selective PPAR $\gamma$ modulator $(5,6,18)$.

Orally ingested, LT glycosides are hydrolyzed by $\beta$-glucosidase to LT and then absorbed into the blood as conjugated metabolites, such as LT glucuronides and sulphates, which have sulfate groups in an arrangement similar to the mono- and disulfates of luteolin seagrass Zostera and are similar species to DSL. Indeed, the free aglycone concentration in circulating blood is undetectably low in humans and animals $(19,20)$. Therefore, luteolin metabolites are presumably responsible for luteolin anti-inflammatory effects. Luteolin glucuronides, particularly luteolin-7-O-glucuronide, have been demonstrated to reduce the expression of inflammatory genes the lipopolysaccharide-treated cells (20).

Should be emphasized that flavanone-class PPAR $\gamma$ partial agonists, luteolin and hesperetin glucuronides, showed similar activation profiles of the PPAR $\gamma$ ligand-binding domain mutants, even though they have different side chain functionalities (21). These glucuronides showed an additive PPAR $\gamma$-transactivating effect with the antidiabetic drug troglitazone, a thiazolidine-2,4-dione which acts as a full PPAR $\gamma$ agonist. The additive effect may indicate that flavanone glucuronides activate PPAR $\gamma$ in a different manner to that of thiazolidine-2,4-dione derivatives. These glucuronides and troglitazone have distinct mechanisms of PPAR $\gamma$ activation, probably due binding to different sites of PPAR $\gamma$. This additive effect may indicate that flavone glucuronides activate PPAR $\gamma$ in a different manner from thiazolidine-2,4-dione derivatives, possibly by binding to the PPAR $\gamma$ ligand-binding domain at a different site (21).

Thus, PPARs are among the main molecular targets of RA, LT and several of its structural analogues and conjugates. TZDs (pioglitazone, rosiglitazone) and fibrates have a selective effect on PPAR $\gamma$, which is a target for the treatment of T2D. PPAR $\gamma$ full agonist TZDs are effective insulin sensitizers and anti-inflammatory agents; however, their use is limited by adverse effects. The major side effects of all TZDs are water retention and, as a consequence, edema. Caution should therefore be taken when TZDs are prescribed and patients should be made aware of the potential water retention/weight gain $(22,23)$. Thus, the search for other effective and safe therapeutic agents remains an urgent issue.

\section{Antiinflammatory and antioxidant activity of RA, LT and its analogues}

Inflammation is considered a potential mechanism in the progression of numerous pathologies, including insulin resistance, cardiovascular diseases and T2D. RA and LT, as main PPC components, modulate inflammatory responses in animal organisms via various mechanisms, a few of which depend on PPARs, whereas others are PPARs-independent but are regulated by numerous transcriptional factors; therefore, the mechanisms of action of these phenolic compounds have a pleiotropic pattern $(19,24,25)$.

In the mechanism of damage and defense associated with oxidative stress, the process of lipid peroxidation (LP) has an important role. In the case of LP, the formation of pathological changes in MS and T2D are predominantly the result of functional impairment of the antioxidant system that seeks to protect the organism. LP syndrome develops upon excessive accumulation of the products of LP in the organism, which includes pathological constituents such as damage to membrane lipids, lipoproteins and proteins, inactivation of enzymes, and impairment of cell division and phagocytosis. These pathological constituents subsequently lead to changes in the structural and functional organization of the membranes and the formation of various pathological processes. Therefore, due to their anti-inflammatory and antioxidant actions, the major components of PPC, RA, LT and its sulfated derivatives, may be utilized for the treatment of MS and T2D $(26,27)$. PPC and its main components showed a high degree of safety and lack of side effects $(8,26)$.

$\mathrm{LT}$ is known to inhibit damage-inducing reactive oxygen species (ROS) and is considered an antioxidant via various mechanisms of action. First, LT functions as a scavenger of ROS through being oxidized by it (24). LT has the structural elements required for antioxidant activity of flavonoids: Hydroxyl moieties at the $3^{\prime}$ and $4^{\prime}$ positions, a double bond between carbon atoms in positions 2 and 3 , and a carbonyl group in position 4 (25). Hydrogen atoms from the hydroxyl groups situated on the aromatic rings can be donated to free radicals. As an aromatic compound, LT can support unpaired electrons around the M-electron system. Direct evidence that LT is a ROS scavenger was obtained from acellular systems (27). Second, LT inhibits ROS-generating oxidases. For instance, LT suppresses the formation of superoxide anions $\left(\mathrm{O}_{2}{ }^{-}\right)$by 
inhibiting the activity of xanthine oxidase (28). However, it remains elusive whether $\mathrm{LT}$ influences ROS formation in the mitochondria of mammalian cells, which are the main site for ROS formation, although it has an effect on the mitochondrial electron transport chain in parasitic (leishmanial) cells (29). Third, LT may exert its antioxidative action by protecting or enhancing endogenous antioxidants such as superoxide dismutase, catalase, glutathione-S-transferase, and glutathione reductase (30). As has been recently shown, LT increased the endogenous mRNA and protein levels of nuclear factor erythroid-2 p45-related factor 2 (Nrf2), which is a critical intracellular regulator in the adaptive response via regulation of a wide array of cytoprotective enzymes, as well as Nrf2 target genes. LT significantly and dose-dependently decreased the production of cytosolic phospholipase A2 (cPLA2), nitric oxide (NO) and inducible NO synthase (iNOS), which were induced by treatment of cells with $1 \mu \mathrm{g} / \mathrm{ml}$ lipopolysaccharide (LPS) for $24 \mathrm{~h}$. Thus, LT significantly activated the phosphoinositide 3-kinase/Nrf2/antioxidant response elements system, and this activation may be responsible for its anti-inflammatory effects, as demonstrated by the suppression of LPS-induced NO, iNOS and cPLA2 (31). Fourth, LT directly inhibits the enzymes that catalyze oxidation of cellular components. For instance, LT suppresses the formation of malondialdehyde in liver lipids, which is stimulated by lipoxygenase (LOX), cyclooxygenase (COX) and ascorbic acid (32). Finally, LT chelates transition metal ions responsible for production of ROS, and therefore inhibits reactions of LOX and suppresses oxidation depending on non-transition metals (30). It should be noted that the LT may also exert its antioxidant effects in vitro. For instance, LT inhibited LPS-induced production of hydroxyl anions $\left({ }^{\circ} \mathrm{OH}\right)$ in macrophages by trapping $\mathrm{O}_{2}{ }^{\cdot-}$ or by inhibiting the activity of xanthine oxidase, or by a combination of the two mechanisms (33).

It is important to emphasize that RA markedly surpasses other known antioxidants, including LT, in tests for antioxidant activity in the systems (hemoglobin- $\mathrm{H}_{2} \mathrm{O}_{2}$-luminol) and [2,2'-azobis(2-methylpropionamidine)dihydrochloride-luminol] and with regard to the inhibition of peroxidation of linoleic acid. According to the level of their antioxidant activity, the studied substances can be arranged in the following order: RA > dihydroquercetin $>$ LT $>$ trolox $>$ ascorbic acid. RA molecules mainly localized in lipid bilayer in the region of polar groups, while not significantly affecting its structural and functional properties, as the permeability of the planar bilayer membranes was not changed by RA at $0.5-10 \mu \mathrm{g} / \mathrm{ml}$. To fully prevent LP, 1 mol\% RA spontaneously incorporated in the lipid bilayer is sufficient. The antioxidant effect of RA is based on its ability to inhibit the initial stage of free radical reactions of LP, much determined by the formation of ROS (34).

The pronounced antioxidant activity of RA has a positive significance, particularly in the progression of oxidative stress in various biological systems, which is considered to be the process of excessive ROS production. The LP process has an important role in the mechanisms of damage and protection during oxidative stress (34). Oxidative stress as well as inflammatory processes are implicated in diabetic vascular complications. By exerting its antioxidant as well as anti-inflammatory effects, RA protects aortic endothelial function and ultrastructure against diabetes-induced damage (35).
LT is also known as an anti-inflammatory and cytoprotective agent. It is successfully used for treating diseases such as T2D, MS and obesity, which are associated with the expansion of chronic inflammatory reactions, which in turn are characterized by an increase in macrophage infiltration, changes in cytokine production and activation of the inflammatory signaling pathway in adipose tissue. In obesity, fatty acid levels, particularly those of saturated free fatty acids, are elevated and directly induce inflammatory responses in macrophages via toll-like receptor 4 (TLR4) and LPS receptor. In numerous cell types, including macrophages, nuclear factor $\kappa \mathrm{B}(\mathrm{NF}-\kappa \mathrm{B})$ and c-Jun N-terminal kinase (JNK) pathways are important modulators of inflammatory gene expression downstream of TLR4. In this way, adipocytes and macrophages interact in a paracrine manner and create a self-amplifying cascade of inflammation that augments the inflammatory changes and insulin resistance in obese adipose tissue (34-39). It is therefore plausible that pharmacological agents capable of stopping the cascade of inflammatory reactions at the molecular level possess antidiabetic properties. In macrophages, LT inhibited the release of the tumor necrosis factor- $\alpha$ (TNF- $\alpha$ ) and activation of NF- $\kappa \mathrm{B}$ induced by LPS, and reduced the mRNA levels TNF- $\alpha$, interleukin-6 (IL-6) and monocyte chemotactic factor-1. Upon stimulation with insulin, LT increased glucose uptake by adipocytes and enhanced the expression of adiponectin and liptin genes; however, the transcriptional activity of PPAR $\gamma$ had the most pronounced effect on the presence of LT $(40,41)$.

Thus, LT reduces insulin resistance in obese adipose tissue; in other words, it acts as an important corrective agent of T2D-associated disorders at the cellular and molecular level. It may therefore be assumed that the main components of PPC, RA and LT, by interfering with the TLR4/NF- $\kappa \mathrm{B}$ and TLR4/JNK axes, may be useful in preventing the onset of insulin resistance in $\mathrm{T} 2 \mathrm{D}$ patients.

It is known that persistent hyperglycemia and increased FFA levels contribute to oxidative stress, which is thought to be the immediate cause of the onset and progression of T2D and its complications (42). Per os administration of RA $(100 \mathrm{mg} / \mathrm{kg}$ ) in a rat model of T2D (high-fat diet and streptozotocin) greatly increased the sensitivity to insulin; at the same time, levels of glucose, glycated hemoglobin, glycation end products, TNF- $\alpha$, IL-1 $\beta$, IL-6, NO, phosphorylated-JNK, P38 mitogen-activated protein kinases and NF- $\mathrm{B}$ in blood plasma significantly declined with a simultaneous rise of the insulin levels in diabetic rats. Furthermore, treatment with RA significantly decreased the levels of triglycerides, FFA, cholesterol and LP in blood plasma and pancreas of rats. The reduced activity of superoxide dismutase, catalase, glutathione peroxidase and glutathione-S-transferase, and also the lower levels of ceruloplasmin, vitamins $\mathrm{C}$ and $\mathrm{E}$, and reduced glutathione in blood plasma of diabetic rats were largely normalized as a result of RA treatment. This indicated a high therapeutic potential of RA, which was confirmed by the increased levels of Nrf2, which is a critical intracellular regulator in adaptation processes via the regulation of a wide array of cytoprotective enzymes (42).

Histological, ultrastructural and immunohistochemical results have demonstrated that oral administration of RA protects pancreatic $\beta$-cells from oxidative stress caused by 


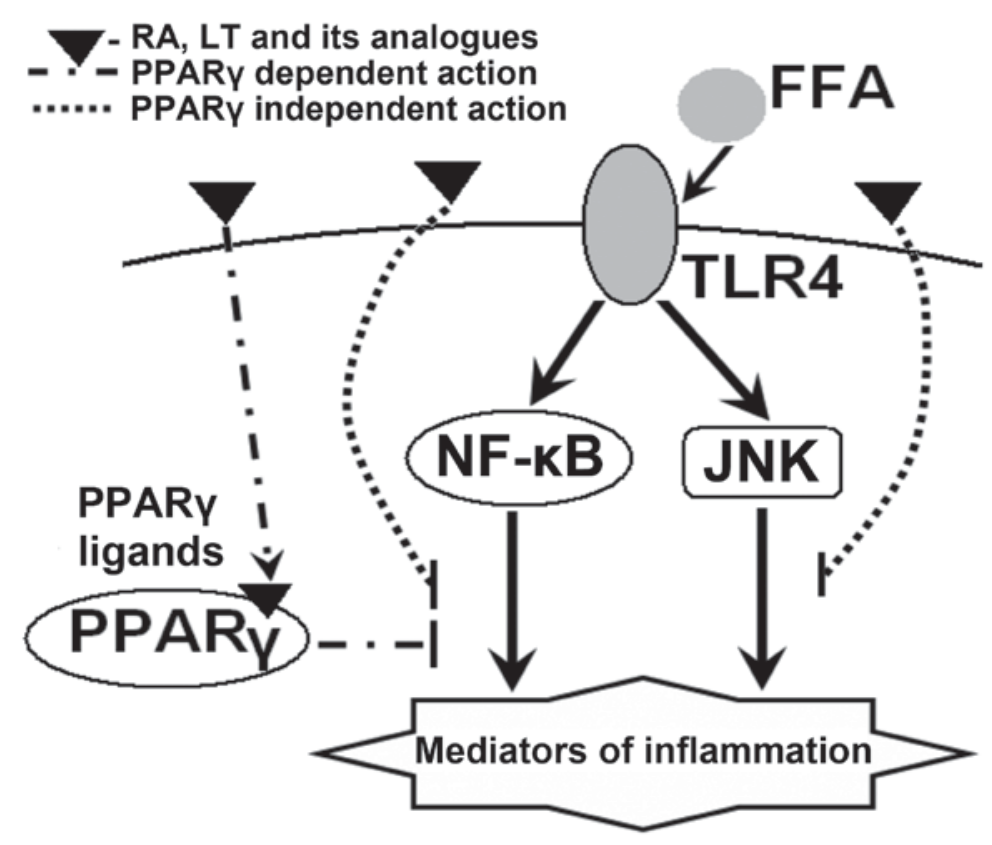

Figure 3. Signaling pathways of inflammatory gene expression in type 2 diabetes and metabolic syndrome and the strategy to prevent these pathologies with RA, LT and its analogues. RA, rosmarinic acid; LT, luteolin; NF, nuclear factor; FFA, free fatty acids; PPAR, peroxisome proliferator-activated receptor; TLR, Toll-like receptor; JNK, c-Jun N-terminal kinase.

high-fat diet and streptozotocin in an experimental model of diabetes. It can be assumed that oral administration of RA relieves the symptoms of diabetes mostly due to its antioxidant properties $(34,43)$.

Fig. 3 depicts a probable pattern of changes in functions and expression of genes participating in inflammatory signaling pathways in diabetes under the effect of RA, LT and its analogues.

Metformin, a pharmaceutical drug most widely used in the treatment of T2D, has a mechanism of action similar to that of LT and RA. Metformin blocks the synthesis of the transcription factor $\mathrm{NF}-\kappa \mathrm{B}$, which controls the downstream synthesis of certain molecules involved in inflammatory responses. The fact that metformin also activates the synthesis of Nrf2, which controls the expression of numerous enzymes involved in the antioxidant protection of the body, is equally important. These enzymes scavenge ROS, thus stopping the activity of macrophages, while their deregulation or loss of function causes mortality of pancreatic $\beta$-cells, resulting in diabetes (44).

It should be noted that besides the action through PPAR-dependent and -independent signaling pathways, RA, LT and its analogues exhibit a pharmacological activity via other intracellular signaling and biochemical pathways: i) They inhibit the formation of enzymes (e.g. PLA2, COX and LOX) involved in the synthesis of eicosanoids, and as a result, the content of proinflammatory molecules (prostaglandins and leukotrienes) declines; ii) they also inhibit factors of transcriptional activation that modulate expression of proinflammatory genes (COX-2, iNOS, TNF- $\alpha$ as well as IL-1 $\beta$ and -6) (4-9,13), as presented in Fig. 4.

The basic mechanisms of these properties are not fully understood, but can partly be explained by the redox properties of these compounds. This means that in various cell systems, they may engage in redox reactions, thus performing functions as donors and acceptors of electrons and protons. The redox properties of RA, LT, and most phenolic compounds are not only confined to their antioxidant and anti-inflammatory activity. Moreover, it was shown that LT exhibits oxidant properties when acting on cells at low concentrations (19). Experimental studies by our group on the combined use of PPC from seagrasses of the genus $Z$. and its main components, RA and DSL, with metformin, based on an alloxan-induced diabetes model, did not reveal any synergistic or additive effects. It is important to emphasize that the protective activity of PPC was higher than that of its main components, RA and DSL (4-7).

A different pattern was observed when physical exercise was combined with the therapeutic use of antioxidants (vitamins $\mathrm{C}$ and $\mathrm{E}$ ) and metformin. A combined therapy of this type appeared to have opposing physiological consequences $(45,46)$. With this regard, it is important to be aware of the molecular mechanisms of action of various substances with anti-diabetic properties when used in combination for the purpose of enhancing their protective effects against the progression of pathological processes; it may also be important to be aware of their action towards normal cells.

Obviously, the mechanisms of modulation of cellular signaling pathways differ between normal and pathological cells. For instance, LT inhibits JNK in macrophages but activates this kinase in tumor cells (47). Furthermore, LT inhibits the activity of $\mathrm{NF}-\kappa \mathrm{B}$ in epithelial cells and macrophages during inflammation by suppressing the activation of inhibitor of $\mathrm{NF}-\kappa \mathrm{B}$, while in tumor cells, participation of $\mathrm{NF}-\kappa \mathrm{B}$ in nuclear events is apparently not suppressed in the presence of LT (48).

It may be assumed that various mechanisms of $\mathrm{NF}-\kappa \mathrm{B}$ suppression exist in normal and pathological cells, which obviously depends on the redox status of these cells and/or on the redox-regulating properties of the active phenolic agent, particularly LT. 


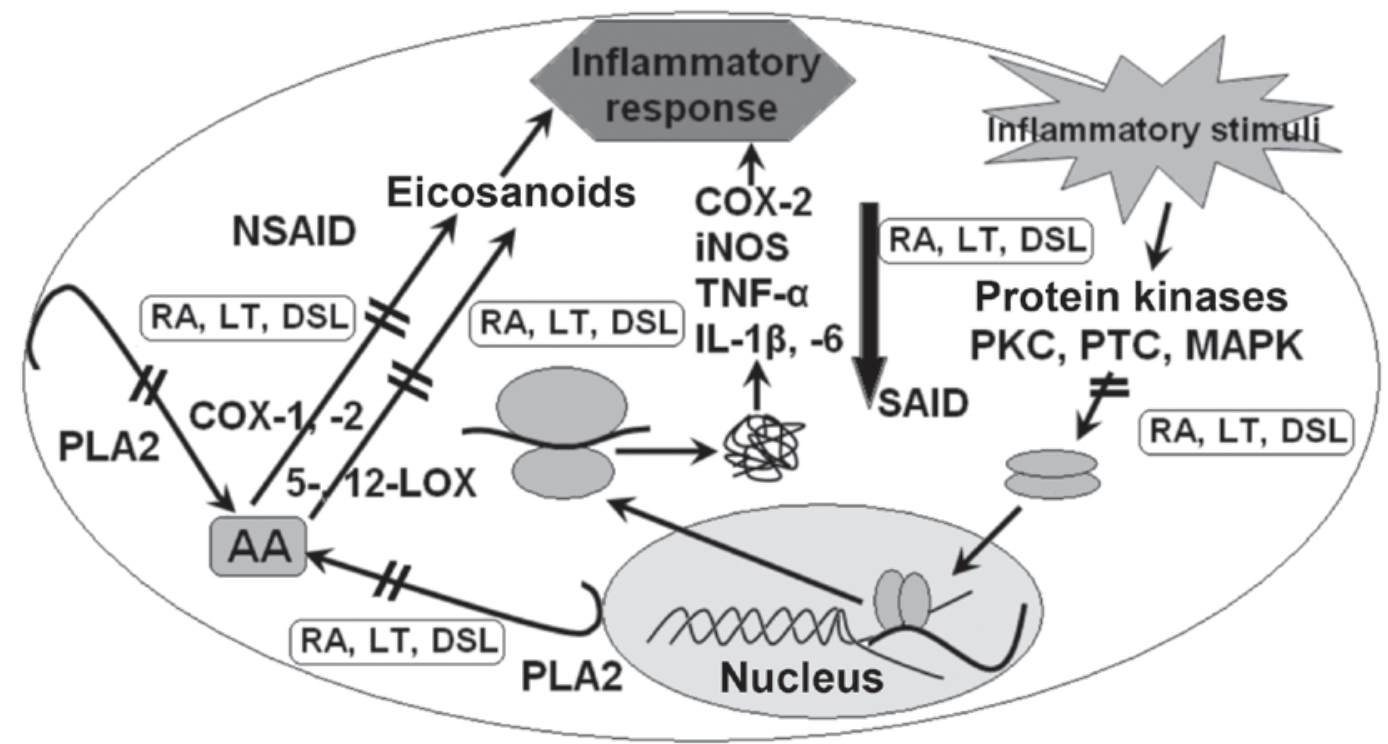

Figure 4. Proposed mechanisms of the AA of RA, LT and DSL. RA, rosmarinic acid; LT, luteolin; DSL, 7,3'-disulfate luteolin; NSAID, non-steroidal anti-inflammatory agent; SAID, steroidal anti-inflammatory agent; PKC, protein kinase C; PTC, protein tyrosine kinase; MAPK, mitogen-activated protein kinase; iNOS, inducible nitric oxide synthase; COX, cyclooxygenase; LOX, lipoxygenase; TNF- $\alpha$, tumor necrosis factor; IL, interleukin; PLA2, phospholipase A2; AA, arachidonic acid.

\section{Features of the antidiabetic action of DSL}

Flavonoids are thought to exhibit a high bioactivity in pure form, but their metabolites are far less active and are mainly the 'deposited' form of these medicinal agents. However, recent findings contradict this dogma. In numerous cases, flavonoids do not lose their activity upon metabolization by processes including sulfation or glycosylation, particularly with regard to their anti-inflammatory and antioxidant potential. Moreover, in certain cases, the effectiveness of the protective action of conjugated forms of phenolic compounds on certain molecular targets in cells and tissues was markedly increased as compared to that of the native phenols, while their cytotoxicity was declined $(8,9,49)$.

Sulfation is an important metabolic pathway of flavonoids in plants. Previous studies by our group found a significant amount of DSL in seagrasses of the $Z$. family (Z. marina and $Z$. asiatica) and developed a simple method to isolate it. These studies showed that in numerous cases, the pharmacological activity of DSL was higher than that of LT (7-10). It may be assumed that DSL is a natural water-soluble form of LT, which is absorbed and reaches the blood plasma of animals and human through the intestine, skipping the stages of modification in intestinal and liver cells. DSL is likely to have a lower toxic potential than LT and thus, the efficiency of its pharmacological action may be increased.

When flavonoids enter the human body, sulfation and glycosylation represent the main ways of their metabolic transformation. It is logical to assume that therapeutic and preventive medications based on flavonoid derivatives, to be developed in the future, are equally promising to non-modified flavonoids that are already widely used in medical practice $(8,9)$.

A study by our group assessing the actions of the sulfated conjugate LT-DSL in models of T2D and hyperlipidemia revealed a higher therapeutic activity than LT (5-9). It may be assumed that, similar to resveratrol (50), sulfated LT is absorbed by epithelial cells more effectively than pure LT. In addition, it is possible that, similar to estrone sulfate (51), sulfated conjugates of LT are an inactive pool of this flavone and can reach target cells upon hydrolysis by sulphatases.

As is known, organic anions of the type of sulfated conjugates of phenolic compounds, with the assistance of designated transporters (symporters) and a significant proton gradient, possess the ability to permeate into intestinal epithelial cells, followed by their rapid transport into blood plasma $(8,52)$. These specific transporters are members of a large family of organic anion transporters and are located at the apical part of membranes of epithelial cells in the small intestine and other organs. They are responsible for transportation of large amounts of anionic organic molecules, which differ structurally from each other.

Our research and analysis of literature data indicates that DSL is able to reach the blood plasma of animals and humans through the intestine, skipping the stages of modification in intestinal and liver cells, which increases its bioabsorbability and effectiveness of its pharmacological action. DSL and LT are likely to interact with the same target receptors and exert a pharmacological effect. DSL serves as an inactive pool of LT and reaches target receptors inside these cells only when hydrolyzed by sulfatases (enzymes of the esterase class that catalyze the hydrolysis of sulfate esters). Fig. 5 shows several variants of interaction of LT and DSL with receptor protein targets on the plasma membrane of macrophage cells and inside these cells.

After entering the bloodstream, DSL and LT begin to interact with endothelial cells of blood vessels, which may be in the normal or a pathological state (e.g. diabetes, hyperlipidemia or atherosclerosis-associated cardiovascular diseases) (Fig. 6A). Fig. 6B depicts the proposed pathways of inhibition of endocytosis of oxidized low-density lipoproteins (oxLDL), mediated by scavenger receptors (SR), in macrophage cells in the presence of LT and DSL. It may be assumed 


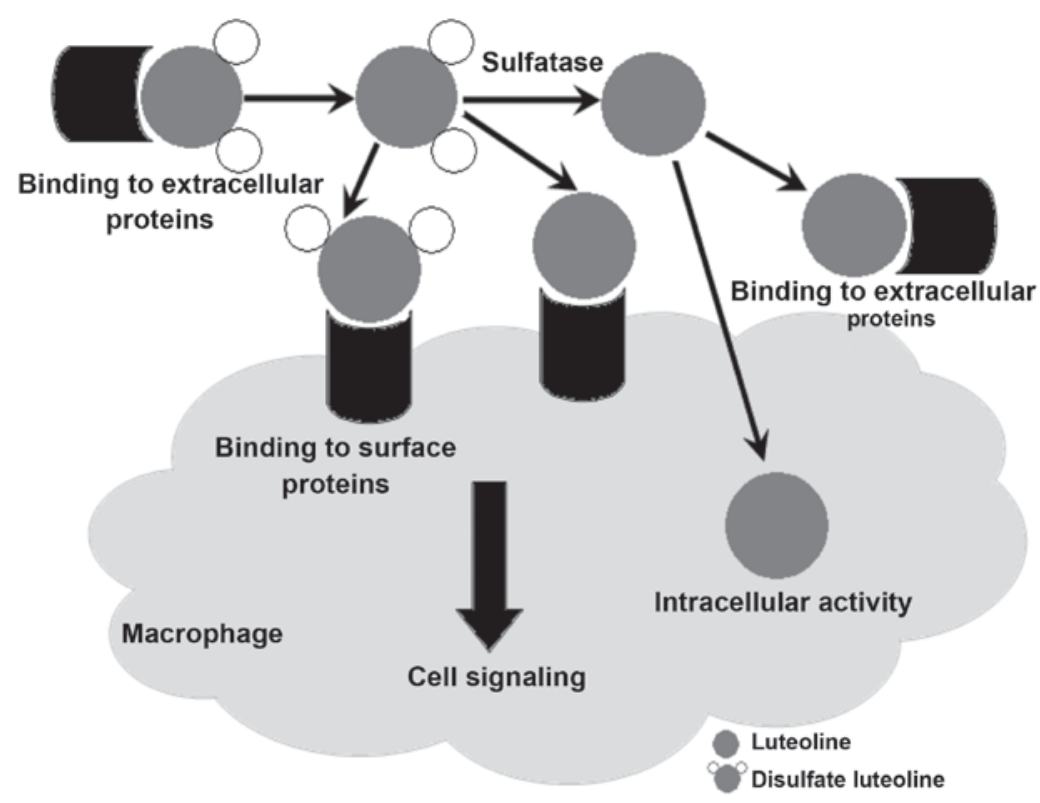

Figure 5. Proposed mechanisms of the protective effects of luteolin and 7,3'-disulfate luteolin on the example of macrophage cells.

\section{A Endothelial cells}

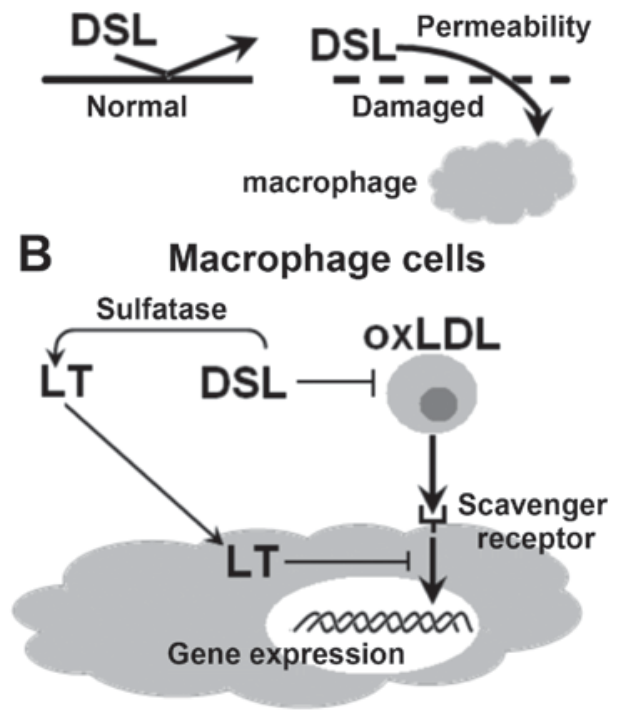

Figure 6. Proposed ways of permeation of LT and DSL into blood vessel cells and their inhibitory effect on scavenger receptors of oxLDL. (A) Permeation of DSL through damaged endothelial cells into the intima, where macrophage cells are located. (B) Inhibitory effect of LT and DSL on permeation of oxLDL into macrophage cells. oxLDL, oxidized low-density lipoproteins; LT, luteolin; DSL, 7,3'-disulfate luteolin.

that the interaction of DSL with SR, localized on the plasma membrane of macrophages, selectively blocks the binding of oxLDL to these receptors and their delivery into the cell. At the early stages of atherosclerosis, SR are considered the most important structural components, which are responsible for the absorption of oxLDL by macrophages, subsequently resulting in the formation of foam cells (precursors of atherosclerotic plaques). Thus, the inhibitory effect of LT on this process may also occur inside a macrophage cell $(8,9)$.

Based on the abovementioned information, the protective action of LT and DSL in the normal state and in the case of cardiovascular diseases and T2D may be divided into several stages: i) Damaged walls of blood vessels allow for entry of metabolites from circulating blood; ii) DSL is safely absorbed and subsequently delivered to target cells; iii) DSL interacts with SR located on macrophages and exerts its the anti-atherosclerotic effects (Fig. 6).

\section{Conclusions}

Inflammation is a systemic protective response of plants, animals and humans to the effect of pathogenic and stress factors of the environment (bacteria, viruses and oxidative stress inducers). In plants, the inflammatory reaction manifests in the form of a hypersensitive response, while presenting as and immune response in animals and humans. Numerous polyphenolic compounds, including RA, LT and DSL, can be considered as endogenous protective means involved in the control of an induced inflammatory process in a producer organism.

Since inflammation has a crucial role in the progression of $\mathrm{T} 2 \mathrm{D}$-associated pathologies, including insulin resistance, cardiovascular diseases, obesity and certain immune disorders, the positive therapeutic effects of PPC from $Z$. seagrasses and its constituents, RA, LT and DSL, in T2D and hyperlipidemia models, are plausible, as outlined in the present review. RA, LT and DSL are used as therapeutic agents exhibit a high antioxidant, anti-inflammatory and immunomodulatory activity. It should be noted that, in spite of the lack of highly selective activity, they have an effect on the functional activity of numerous cell targets and signaling pathways with multiple mechanisms of action by regulating the progression of various inflammatory reactions through direct and indirect effects on ROS via PPAR-dependent and -independent pathways.

When used for therapeutic purposes, RA, LT and DSL have unique features regarding the mechanism of their anti-inflammatory and antioxidant activity; of note, PPC exerted a more 
pronounced pharmacological effect than its major components, RA, LT and DSL, applied separately. For this reason, the use of combined pharmaceutical agents as PPC $(5,26)$, acting on several different targets in cells, appears to be a promising approach for development of remedies of complementary therapy for preventing and treating multifactorial metabolic diseases. This approach may overcome the abovementioned problems regarding undesirable side effects of potent synthetic PPAR agonists. Future studies are required to further clarify the mechanisms of action of RA, LT and DSL in T2D and associated diseases.

Clarification of the 'intricate pattern' of redox reactions and features of interaction of RA, LT and DSL with various cellular targets in normal and pathological cells and tissues, depending on factors including their dose, cell type and pathology, may provide more effective antidiabetic therapies. For this purpose, the features and functioning of biochemical signaling pathways and their interactions should be fully elucidated. Understanding the molecular mechanisms of their antidiabetic action will facilitate the effective application of RA, LT and DSL for the prevention and treatment of T2D along with the widely used modern pharmaceutical drugs.

\section{Acknowledgments}

This study was supported by the grant Far Eastern Branch of the Russian Academy of Sciences (grant no. N15-I-5-018).

\section{References}

1. Finucane MM, Danaei G and Ezzati M: Bayesian estimation of population-level trends in measures of health status. Statistical Sci 29: 18-25, 2014.

2. PalomerX,BarrosoE,Zarei M,Botteri G and Vázquez-Carrera M: 2PPAR $\beta / \delta$ and lipid metabolism in the heart. Biochim Biophys Acta 1860: 1569-1578, 2016.

3. Tuttolomondo A, Maida C and Pinto A: Diabetic foot syndrome: Immune-inflammatory features as possible cardiovascular markers in diabetes. World J Orthop 6: 62-76, 2015.

4. Krylova NV, Popov AM and Leonova GN: Antioxidants as potential antiviral agents for flavivirus infections (Review). Antibiotiki i Khimioterapiya 61: 25-31, 2016 (In Russian)

5. Krivoshapko ON, Popov AM, Artyukov AA and Kostetskiı EIa: Features of corrective actions polar lipids from marine and aquatic bioantioxidants in disorders of lipid and carbohydrate metabolism. Biomed Khim 58: 189-198, 2012 (In Russian).

6. Krivoshapko ON and Popov AM: Curative and preventive properties of lipids and antioxidants isolated from marine hydrobionts. Vopr Pitan 80: 4-8, 2011 (In Russian).

7. Popov AM and Krivoshapko ON: Protective Effects of Polar Lipids and Redox-active compounds from marine organisms at modeling of hyperlipidemia and diabetes. J Biomed Sci Eng 6: 543-550, 2013.

8. Popov AM, Krivoshapko ON and Artyukov AA: Protective mechanisms of the pharmacological activity of flavonoids. Rus J Biopharm 4: 27-41, 2012.

9. Popov AM, Krivoshapko ON and Artyukov AA: Comparative evaluation of the pharmacological activity of luteolin and luteolin 7,3'-disulfate in modeling different pathologies. Rus J Biopharm 3: 27-33, 2011.

10. Popov AM, Artyukov AA, Krivoshapko ON, Krylova NV, Leonova GN and Kozlovskaja EP: Medication, processing antioxidant, cardioprotective, antidiabetic, anti-inflammatory, hepatoprotective, antitumoral and antiviral action. Patent Russian Federation 2432959, 2011.

11. Gervois P, Fruchart JC and Staels B: Drug Insight: Mechanisms of action and therapeutic applications for agonists of peroxisome proliferator-activated receptors. Nat Clin Pract Endocrinol Metab 3: 145-156, 2007.
12. Mueller M, Lukas B, Novak J, Simoncini T, Genazzani AR and Janqbauer A: Oregano: A source for peroxisome proliferator-activated receptor gamma antagonists. J Agric Food Chem 56: 11621-11630, 2008

13. Tsybulsky AV, Popov AM and Artyukov AA: The Comparative study of the medical action of lyuteolin, rosmarinic acid and echinochrome A at experimental stress-induced cardiovascular. Biomed Khim 57: 314-325, 2011.

14. Liu ZM, Hu M, Chan P and Tomlinson B: Early investigational drugs targeting PPAR- $\alpha$ for the treatment of metabolic disease. Expert Opin Investig Drugs 24: 611-621, 2015.

15. Xagorari A, Papapetropoulos A, Mauromatis A, Economou M, Fotsis T and Roussos C: Luteolin inhibits an endotoxin-stimulated phosphorylation cascade and proinflammatory cytokine production in macrophages. J Pharmacol Exp Ther 296: 181-187, 2001.

16. Ding L, Jin D and Chen X: Luteolin enhances insulin sensitivity via activation of PPAR $\gamma$ transcriptional activity in adipocytes. J Nutr Biochem 21: 941-947, 2010.

17. Park HS, Kim SH, Kim YS, Ryu SY, Hwang JT, Yang HJ, Kim GH, Kwon DY and Kim MS: Luteolin inhibits adipogenic differentiation by regulating PPARgamma activation. Biofactors 35: 373-379, 2009.

18. Puhl AC, Bernardes A, Silveira RL, Yuan J, Campos JL, Saidemberg DM, Palma MS, Cvoro A, Ayers SD, Webb P, et al: Mode of peroxisome proliferator-activated receptor $\gamma$ activation by luteolin. Mol Pharmacol 81: 788-799, 2012.

19. Lopez-Lázaro M: Distribution and biological activities of the flavonoid luteolin. Mini Rev Med Chem 9: 31-59, 2009.

20. Kure A, Nakagawa K, Kondo M, Kato S, Kimura F, Watanabe A, Shoji N, Hatanaka S, Tsushida T and Miyazawa T: Metabolic fate of Luteolin in rats: Its relationship to anti-inflammatory effect. J Agric Food Chem 64: 4246-4254, 2016.

21. Gamo K, Shiraki T, Matsuura N and Miyachi H: Mechanism of peroxisome proliferator-activated receptor gamma (PPAR $\gamma)$ transactivation by hesperetin glucuronides is distinct from that by a thiazolidine-2,4-dione agent. Chem Pharm Bull (Tokyo) 62: 491-493, 2014.

22. Weidner C, Wowro SJ, Freiwald A, Kodelja V, Abdel-Aziz H, Kelber O and Sauer S: Lemon balm extract causes potent antihyperglycemic and antihyperlipidemic effects in insulin-resistant obese mice. Mol Nutr Food Res 58: 903-907, 2014.

23. Molavi B, Rassouli N, Bagwe S and Rasouli N: A review of thiazolidinediones and metformin in the treatment of type 2 diabetes with focus on cardiovascular complications. Vasc Health Risk Manag 3: 967-973, 2007.

24. Lien EJ, Ren S, Bui HH and Wang R: Quantitative structure-activity relationship analysis of phenolic antioxidants. Free Radic Biol Med 26: 285-294, 1999.

25. Nimnual AS, Taylor LJ and Bar-Sagi D: Redox-dependent downregulation of Rho by Rac. Nat Cell Biol 5: 236-241, 2003.

26. Popov AM, Krivoshapko ON, Klimovich AA and Artyukov AA: Biological activity and mechanisms of therapeutic action of rosmarinic acid, luteolin and its sulphated derivatives. Biomed Khim 62: 22-30, 2016 (In Russian).

27. Popov AM, Osipov AN, Korepanova EA, Krivoshapko ON, Artyukov AA and Klimovich AA: Study of antioxidant and membrane activity of luteoline using different model systems. Biophysics (Russian Federation) 61: 1079-1087, 2016.

28. Nagao A, Seki M and Kobayashi H: Inhibition of xanthine oxidase by flavonoids. Biosci Biotechnol Biochem 63: 1787-1790, 1999.

29. Sen N, Das BB, Ganguly A, Banerjee B, Sen T and Majumder HK: Leishmania donovani: Intracellular ATP level regulates apoptosis-like death in luteolin induced dyskinetoplastid cells. Exp Parasitol 114: 204-214, 2006

30. Manju V and Nalini N: Chemopreventive potential of luteolin during colon carcinogenesis induced by 1,2-dimethylhydrazine. Ital J Biochem 54: 268-275, 2005.

31. Robak J, Shridi F, Wolbís M and Królikowska M: Screening of the influence of flavonoids on lipoxygenase and cyclooxygenase activity, as well as on nonenzymic lipid oxidation. Pol J Pharmacol Pharm 40: 451-458, 1988.

32. Paredes-Gonzalez X, Fuentes F, Jeffery S, Saw CL, Shu L, Su ZY and Kong AN: Induction of Nrf2-mediated gene expression by dietary phytochemical flavones apigenin and luteolin. Biopharm Drug Dispos 36: 440-451, 2015.

33. Brown JE and Rice-Evans CA: Luteolin-rich artichoke extract protects low density lipoprotein from oxidation in vitro. Free Radic Res 29: 247-255, 1988. 
34. Popov AM, Osipov AN, Korepanova EA, Krivoshapko ON and Artyukov AA: Study of antioxidant and membrane activity of rosmarinic acid using different model systems. Biofizika 58: 775-785, 2013 (In Russian).

35. Sotnikova R, Okruhlicova L, Vlkovicova J, Navarova J, Gajdacova B, Pivackova L, Fialova S and Krenek P: Rosmarinic acid administration attenuates diabetes-induced vascular dysfunction of the rat aorta. J Pharm Pharmacol 65: 713-723, 2013.

36. Harris GK, Qian Y, Leonard SS, Sbarra DC and Shi X: Luteolin and chrysin differentially inhibit cyclooxygenase-2 expression and scavenge reactive oxygen species but similarly inhibit prostaglandin-E2 formation in RAW 264.7 cells. J Nutr 136: $1517-1521,2006$

37. Fadel O, El Kirat K and Morandat S: The natural antioxidant rosmarinic acid spontaneously penetrates membranes to inhibit lipid peroxidation in situ. Biochim Biophys Acta 1808: 2973-2980, 2011.

38. Kawai Y: Immunochemical detection of food-derived polyphenols in the aorta: Macrophages as a major target underlying the anti-atherosclerotic activity of polyphenols. Biosci Biotechnol Biochem 75: 609-617, 2011.

39. Lee J, Jung E, Kim Y, Lee J, Park J, Hong S, Hyun CG, Park D and Kim YS: Rosmarinic acid as a downstream inhibitor of IKK-beta in TNF-alpha-induced upregulation of CCL11 and CCR3. J Pharmacol 148: 366-375, 2006.

40. Ando C, Takahashi N, Hirai S, Nishimura K, Lin S, Uemura T, Goto T, Yu R, Nakagami J, Murakami S and Kawada T: Luteolin, a food-derived flavonoid, suppresses adipocyte-dependent activation of macrophages by inhibiting JNK activation. FEBS Lett 583: 3649-3654, 2009.

41. Hirai S, Takahashi N, Goto T, Lin S, Uemura T, Yu R and Kawada T: Functional food targeting the regulation of obesity-induced inflammatory responses and pathologies. Mediators Inflamm 2010: 367838, 2010.

42. Köhle C and Bock KW: Activation of coupled Ah receptor and Nrf2 gene batteries by dietary phytochemicals in relation to chemoprevention. Biochem Pharmacol 72: 795-805, 2006.
43. Govindaraj J and Sorimuthu Pillai S: Rosmarinic acid modulates the antioxidant status and protects pancreatic tissues from glucolipotoxicity mediated oxidative stress in high-fat diet: Streptozotocin-induced diabetic rats. Mol Cell Biochem 404: $143-159,2015$.

44. Hirsch HA, Ilipoulos D and Struhl K: Metformin inhibits the inflammatory response associated with cellular transformation and cancer stem cell growth. Proc Natl Acad Sci USA 110: 972-977, 2013

45. Watson JD: Types diabetes as a redox disease. Lancet 383: 841-843, 2014.

46. Ristow M, Zarse K, Oberbach A, Klöting N, Birringer M, Kiehntopf M, Stumvoll M, Kahn CR and Blüher M: Antioxidants prevent health-promoting effects of physical exercise in humans. Proc Natl Acad Sci USA 106: 8665-8670, 2009.

47. Shi RX, Ong CN and Shen HM: Luteolin sensitizes tumor necrosis factor-alpha-induced apoptosis in human tumor cells. Oncogene 23: 7712-7721, 2004.

48. Ju W, Wang X, Shi H, Chen W, Belinsky SA and Lin Y: A critical role of luteolin-induced reactive oxygen species in blockage of tumor necrosis factor-activated nuclear factor-kappaB pathway and sensitization of apoptosis in lung cancer cells. Mol Pharmacol 71: 1381-1388, 2007.

49. Mukinda JT, Syce JA, Fisher D and Meyer M: Effect of the Plant Matrix on the Uptake of Luteolin Derivatives-containing Artemisia afra Aqueous-extract in Caco-2 cells. J Ethnopharmacol 130: 439-449, 2010.

50. Hoshino J, Park EJ, Kondratyuk TP, Marler L, Pezzuto JM, van Breemen RB, Mo S, Li Y and Cushman M: Selective synthesis and biological evaluation of sulfate-conjugated resveratrol metabolites. J Med Chem 53: 5033-5043, 2010.

51. Santner SJ, Feil PD and Santen RJ: In situ estrogen production via estrone sulphatase pathway in breast tumours: Relative importance versus the aromatase pathway. J Clin Endocrinol Metab 59: 29-33, 1984.

52. Burckhardt $\mathrm{G}$ and Burckhardt $\mathrm{BC}$ : In vitro and in vivo evidence of the importance of organic anion transporters (OATs) in drug therapy. Handb Exp Pharmacol 29-104, 2011. 